\title{
Performance Evaluation of Modified SLM Technique in OFDM System Using Selected Codeword Shift
}

\author{
Ezmin Abdullah ${ }^{1}$, Azlina Idris ${ }^{1}$ and Azilah Saparon ${ }^{2}$ \\ ${ }^{1}$ Wireless Communication Technology Group (WiCoT), Faculty Electrical Engineering, UniversitiTeknologi Mara,40450, Shah Alam, \\ Selangor DE, Malaysia \\ ${ }^{2}$ Faculty Electrical Engineering, UniversitiTeknologi Mara,40450, Shah Alam, Selangor DE, Malaysia
}

\begin{abstract}
Selective Mapping (SLM) is a well-known technique used in OFDM System to reduce high Peak-Average Power Ratio (PAPR). High PAPR occurred during signal processing which multiple data are converted into electromagnetic signal via Inverse Fast Fourier transform (IFFT) at the transmitter. Even though SLM technique has a potential to reduce high PAPR, it disrupts the overall performance of bit error rate (BER) at receiver part. In this paper, a modified SLM has been proposed using circulant shift and embedded bit side information to overcome the BER performance degradation at the receiver part as well as to further improve the PAPR performance at the transmitter part. The results have shown an encouraging improvement of PAPR and BER to the OFDM system performance.
\end{abstract}

\section{Introduction}

The demands of mobile communications have progressively led to global energy consumption around the world. Power amplification process contributes to about $50 \%$ of power consumption distribution in radio access technology. In this level, the main component to reduce power consumption relies on the High-Power Amplifier (HPA) efficiency [1].

The efficiency of HPA in multicarrier modulation systems such as OFDM system is associated to the PeakAverage Power Ratio (PAPR) which occurred during the modulation process via Inverse Fast Fourier Transform (IFFT). High PAPR causes power deficiency in the RF amplifier, signal distortion and energy spilling to adjacent channel. The drawbacks will lead to the cost of extending higher range of RF amplifier [2], [3] and computational complexity [4].

Selective Mapping (SLM) technique is widely used in OFDM system in order to reduce high PAPR because of its advantage of avoiding signal distortion [5]. However, SLM technique requires a side information (SI) transmitted together with the signal to allow recovery of original data. In many cases, the erroneous of the SI will cause a confusion of in detecting the correct SI. This problem could affect BER performance [6], [7]. The BER problem can be resolved by adding forward error check (FEC) encoder and decoder at the transmitter and receiver respectively as proposed by Wen [8] et al. However, this application will increase the complexity of the system as well as its cost.
In this paper, a modified SLM technique has been proposed to overcome the BER drawback in SLM as well to further improve the PAPR performance. This technique applied a circulant shift onto parallel bit data and embedded bit SI before digital modulation process in transmitter. The results shows the comparison of PAPR and BER performance between modified SLM, Method 1 of Data Position Permutation (DPP)[8] and Selective Codeword Shift (SCS).

\section{Selective mapping (SLM) technique}

In the conventional Selective Mapping (SLM) technique as seen in Fig. 1, after undergone digital modulation, $u$ number of OFDM symbol candidates are organized in parallel. Every candidate hold a same OFDM symbol sequences, $\quad X=[X(0), X(1), \ldots X(N-1))]^{T} \quad$.The multiplication of every candidate with phase factor, $\mathrm{P}^{u}=\left[\mathrm{P}^{0}, \mathrm{P}^{1}, \ldots, \mathrm{P}^{U-1}\right], 0 \leq u \leq U-1$ will produce $u$ number of alternative OFDM symbol sequences. The alternative symbol sequences can be described as

$$
\mathrm{X}^{u}=\prod_{n=1}^{N} X_{n} \otimes \mathrm{P}^{u}, \quad 0 \leq u \leq U-1
$$

where $\otimes$ denotes the component-wise multiplication of two vectors. The phase sequence, $\mathrm{P}^{u}$ is generated by using a complex number magnitude, $\mathrm{P}^{u}=e^{j \varphi_{u}}$ where $\varphi_{u} \in[0,2 \pi]$. These alternative input sequences, $X^{u}$ are then undergone Inverse Fourier Transform (IFFT) 
process to generate OFDM signal. The signal in the time domain is written as

$$
x^{u}(t)=\frac{1}{\sqrt{N}} \sum_{k=0}^{N-1} \mathrm{X}^{u} \cdot e^{j 2 \pi f_{k} t}, \quad 0<t<N \mathrm{~T}
$$

where $e^{j 2 \pi f_{k} t}$ is the orthogonal series of sinusoids performed by IFFT. Finally, the minimum Peak-Average Power Ratio (PAPR) OFDM signalscandidate will be selected for transmission. The transmitted signals are obtained by

$$
s^{u}(t)=\underset{0 \leq u \leq U-1}{\operatorname{argmin}}\left\{\frac{\max \left|x^{u}(t)\right|^{2}}{E\left|x^{u}(t)\right|^{2}}\right\}
$$

Side information (SI) is embedded together with the transmitted signal in order to decode the received signal at the receiver. The erroneous of the received SI cause the confusion to detect the correct SI index that resulting BER performance degradation.

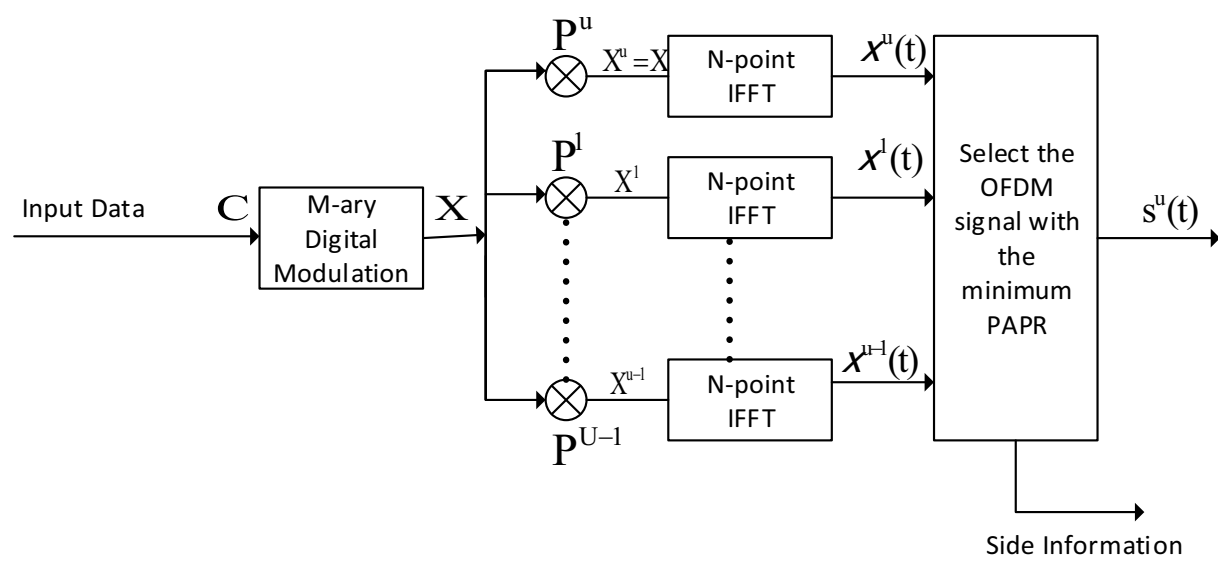

Figure 1. Block diagram of the conventional selective mapping (SLM) OFDM system

\section{Modified selective mapping (SLM)}

A modified Selective Mapping using circulant shift is proposed in this paper, and it shows to have an encouragingPeak-Average Power Ratio (PAPR) reduction performance as compared to conventional SLM.

Based on Eq. (2), Orthogonal Frequency Division Multiplexing (OFDM) signals are generated by multiplication of data symbol $X_{n}$ and orthogonal sinusoids IFFT, $e^{j 2 \pi f_{k} t}$. These two components, $X_{n}$ and $e^{j 2 \pi f_{k} t}$ are the important parts to determine PAPR as power symbols and calculated below

$$
\mathrm{X}_{\text {pow }}^{S}=\frac{\left|\mathrm{X}^{S}\right|^{2}}{2}
$$

Data symbol sequences $X_{n}$ are the complex form of data modulated by the digital modulation QAM to the codeword bits.In this paper, we proposed to manipulate codeword bits, $\mathrm{C}$ using circulant shift (Fig. 2). This technique is limited to the M-ary modulation scheme as the circulant shift will need two or more bits in a codeword to shift the bits in parallel.

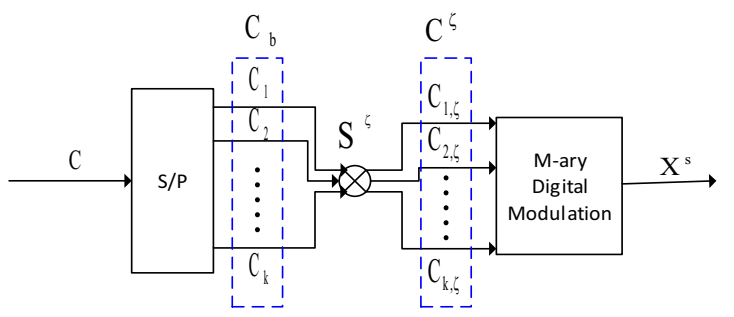

Figure 2. Circulant shift of codeword sub-blocks for one shift factor.
In order to achieve a large PAPR reduction, it is proposed for an increase in the probability of various alternative symbol sequences, $X_{n}$. This technique is taking place at the bits data before digital modulation process. As illustrated in Fig. 2, the bits data, $\mathrm{C}$ is indicated as $\left.\mathrm{c}_{1}, \mathrm{c}_{2}, \ldots, \mathrm{c}_{d}\right]$ and divided into sub-blocks, $\mathrm{C}_{b}=\left[C_{1}, C_{2}, \ldots . C_{K}\right]$, where $=\frac{d}{m}$, is the number of subblock and $m=\log _{2}$ (M-ary QAM), is the number of bits per symbol. Codeword $\mathrm{C}$ in sub-block is represented as

$$
\mathrm{C}_{b}=\prod_{k=1}^{K} C_{k}, \quad 1 \leq k \leq K
$$

where $C_{1}=\left(c_{1}, \ldots, c_{m}\right), C_{2}=\left(c_{m+1}, \ldots, c_{2 m}\right)$ and so on until $C_{K}$ are arranged in parallel.

The modification of SLM is started when circulant shift (one shift to the right) is applied to every sub-block of the parallel bits data. Thus, the alternative codewords are represented as

$$
C^{\zeta}=\prod_{k=1}^{K} C_{k} \otimes S^{\zeta} \quad 0 \leq \zeta \leq m-1
$$

Codeword shift sequences become $C^{\zeta}=\left[C_{1} \otimes\right.$ $\left.S^{\zeta}, C_{2} \otimes S^{\zeta}, \ldots, C_{K} \otimes S^{\zeta}\right]=\left[C_{1, \zeta}, C_{2, \zeta}, \ldots, C_{K, \zeta}\right]$. The codeword shift sequences are then modulated by M-ary digital modulation. The outputs of the symbol sequences are $\mathrm{X}^{S}=\left[\mathrm{X}_{1, \zeta}, \mathrm{X}_{2, \zeta}, \ldots, X_{K, \zeta}\right]$. The process afterward follows the conventional SLM which is illustrated in Fig. 
3. The new alternatives OFDM symbol for modified SLM is given by

$$
\mathrm{X}^{S, u}=\prod_{k=1}^{K} \mathrm{X}_{k}^{S} \otimes \mathrm{P}^{u}, \quad 0 \leq u \leq U-1
$$

Therefore, the alternative OFDM signals in the time domain is represented as

$$
x^{S, u}(t)=\frac{1}{\sqrt{N}} \sum_{k=0}^{N-1} \mathrm{X}^{S, u} \cdot e^{j 2 \pi f_{k} t}
$$

Finally, the minimum PAPR alternative OFDM signals are obtained by

$$
s^{S, u}(t)=\underset{0 \leq u \leq U-1}{\operatorname{argmin}}\left\{\frac{\left.\max \mid x^{S, u}(t)\right)\left.\right|^{2}}{E\left|x^{S, u}(t)\right|^{2}}\right\}
$$

In this paper, the number of $U$ for the phase factor in SLM part is limited where it is based on the number of bit per symbol, $m$. It can be noted that side information of $s^{S, u}$ index is included together in the transmitted signals in order to properly retrieve the data at the receiver.

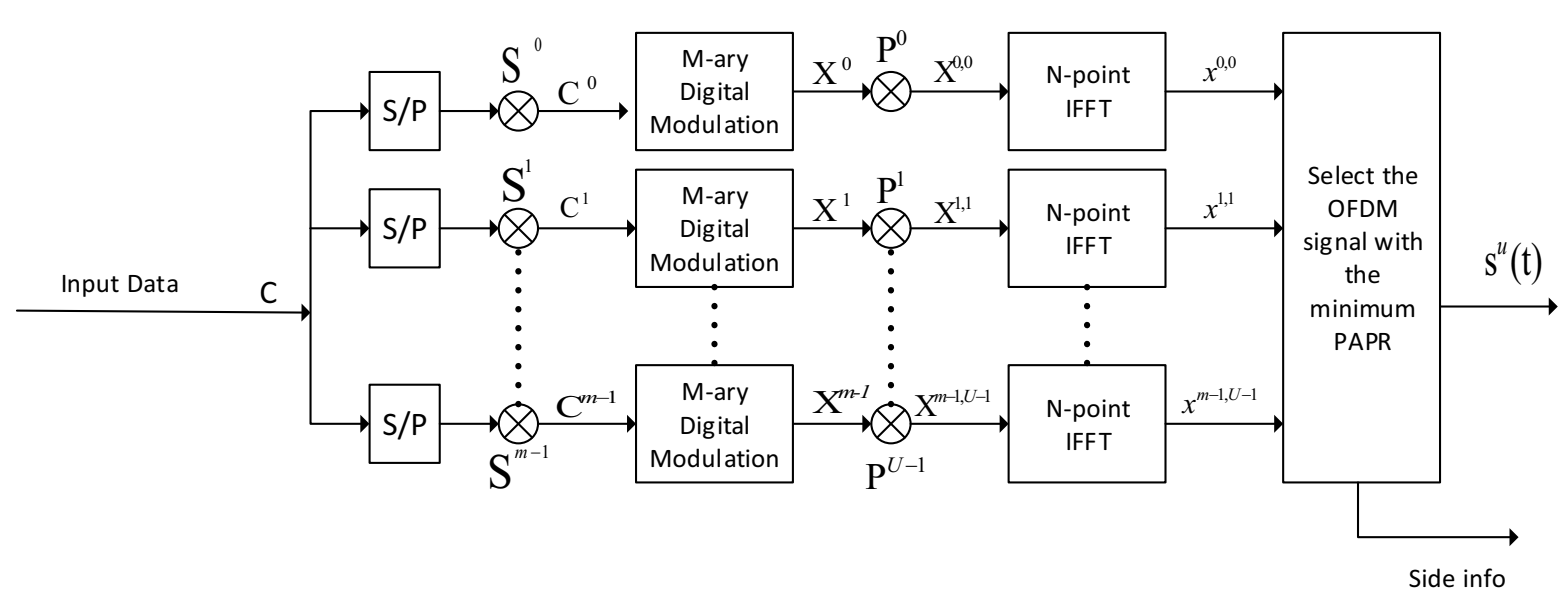

Figure 3. Modified selective mapping (SLM) OFDM system

\subsection{Embedded Bit Side Information (SI)}

Side information (SI) is an important index which is transmitted together with the transmission signal in order to retrieve the original data at the receiver. The index indicates which candidate has been selected for transmission. At the receiver, the data is retrieved based on the information from the SI index.

An embedded bits SI has been developed in this paper. The SI is embedded in the form of binary bits. The positions of $m$ bits of binary SI are situated at the head and at the middle of the codeword block as shown in Fig. 4.

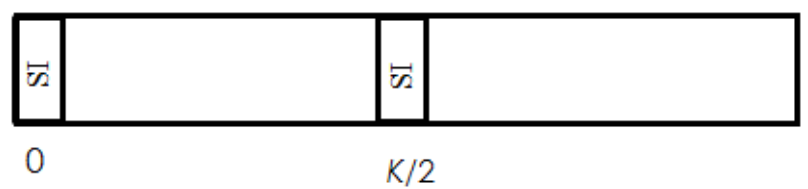

Figure 4. Codeword blocks format with embedded bits side information

The embedded SI can be illustrated as

$C_{1}=\left[\mathrm{SI}(m\right.$ bits $), \mathrm{c}_{1}, \mathrm{c}_{2}, \mathrm{c}_{3}, \mathrm{c}_{4}, \mathrm{c}_{5}, \mathrm{c}_{6}, \mathrm{c}_{7}, \mathrm{c}_{8}, \mathrm{c}_{9}, \mathrm{SI}(m$ bits $), \mathrm{c}_{10}$,

$C_{2}=\left[\operatorname{SI}(m\right.$ bits $), \mathrm{c}_{6}, \mathrm{c}_{1}, \mathrm{c}_{2}, \mathrm{c}_{3}, \mathrm{c}_{4}, \mathrm{c}_{5}, \mathrm{c}_{12}, \mathrm{c}_{7}, \mathrm{c}_{8}, \mathrm{SI}(m$ bits $), \mathrm{c}_{9}$,

$C_{3}=\left[\operatorname{SI}(m\right.$ bits $), \mathrm{c}_{5}, \mathrm{c}_{6}, \mathrm{c}_{1}, \mathrm{c}_{2}, \mathrm{c}_{3}, \mathrm{c}_{4}, \mathrm{c}_{11}, \mathrm{c}_{12}, \mathrm{c}_{7}, \mathrm{SI}(m$ bits $), \mathrm{c}_{8}$,

$C_{4}=\left[\operatorname{SI}(m\right.$ bits $), \mathrm{c}_{4}, \mathrm{c}_{5}, \mathrm{c}_{6}, \mathrm{c}_{1}, \mathrm{c}_{2}, \mathrm{c}_{3}, \mathrm{c}_{10}, \mathrm{c}_{11}, \mathrm{c}_{12}, \operatorname{SI}(m$ bits $), \mathrm{c}_{7}$,

$C_{5}=\left[\operatorname{SI}(m\right.$ bits $), \mathrm{c}_{3}, \mathrm{c}_{4}, \mathrm{c}_{5}, \mathrm{c}_{6}, \mathrm{c}_{1}, \mathrm{c}_{2}, \mathrm{c}_{9}, \mathrm{c}_{10}, \mathrm{c}_{11}, \mathrm{SI}(m$ bits $), \mathrm{c}_{1}$
$C_{6}=\left[\operatorname{SI}(m\right.$ bits $), \mathrm{c}_{2}, \mathrm{c}_{3}, \mathrm{c}_{4}, \mathrm{c}_{5}, \mathrm{c}_{6}, \mathrm{c}_{1}, \mathrm{c}_{8}, \mathrm{c}_{9}, \mathrm{c}_{10}, \operatorname{SI}(m$ bits $), \mathrm{c}_{11}$

\section{Results and discussions}

The performance evaluation of proposed technique has been validated and compared by computer simulations using Matlab.The OFDM system parameters used in the simulation are based on $3^{\text {rd }}$ Generation Partnership Project Long Term Evolution (3GPP-LTE) System [9] standard as shown in Table 1 . Therewere $10^{4}$ of OFDM signals have been considered to calculate the PAPR complementary cumulative distribution functions (CCDF). The performance evaluation consists of modified SLM, conventional SLM and method 1 of DPP are shown in PAPR CCDF and BER graph.To make the computational complexity to be equal, the number of IFFT used in SLM is set to be the same as the number of IFFT used in the modified SLM.

As shown in Fig. 5, the PAPR performance of the modified SLM is effectively reduced the PAPR by about $2.9 \mathrm{~dB}$ from the original OFDM. The modified SLM outperformed the conventional SLM, DPP and SCS with $27.6 \%$ improvement as compared to the SLM, DPP and SCS, $8.6 \%, 12.4 \%$ and $19.0 \%$ respectively. The number of IFFTs block was set to be equal in every reduction technique by following the proposed method minimum system which is $m=6$. Table 2 summarizes the results in Fig. 5. 
Table 1. Simulation parameters for the $3^{\text {rd }}$ Generation partnership project long term evolution (3GPP-LTE) system [9].

\begin{tabular}{cc}
\hline Parameter & Value \\
\hline Bandwidth (BW) & $1.25 \mathrm{MHz}$ \\
Sampling frequency & $1.92 \mathrm{MHz}$ \\
Sampling time & $5.208 \times 10^{-7} \mathrm{sec}$ \\
IFFT size & 128 \\
Used subcarrier & 76 \\
Modulation technique & 64 QAM \\
Guard interval & $1 / 4$ \\
Chanel model & Rayleigh Fading \\
\hline
\end{tabular}

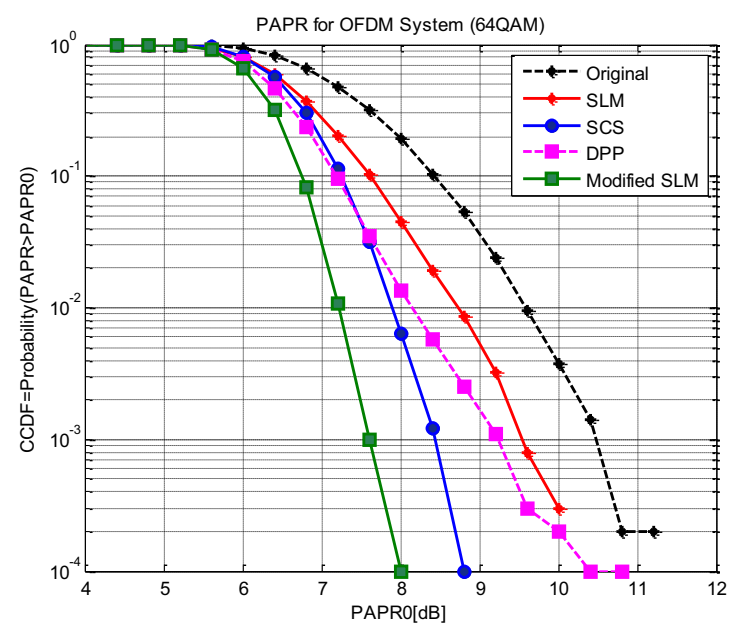

Figure 5. CCDF of the PAPR Modified Selective Mapping (SLM) as compared to original OFDM signal, conventional Selective Mapping, Selected Codeword Shift (SCS) and Data Position Permutation (DPP).

Table 2. PAPR analysis for various PAPR reduction techniques at clip rate $10^{-3}$

\begin{tabular}{ccc}
\hline $\begin{array}{c}\text { OFDM System } \\
\text { (64 QAM, 128IFFT) }\end{array}$ & PAPR in dB & Improvement (\%) \\
\hline Original OFDM & 10.5 & - \\
SLM & 9.6 & 8.6 \\
DPP & 9.2 & 12.4 \\
SCS & 8.5 & 19.0 \\
Modified SLM & 7.6 & 27.6 \\
\hline
\end{tabular}

The BER performance of the system is very important in order to evaluate its robustness. Taking that into account, the investigation of BER performance was conducted in the Rayleigh fading channel and the result are shown in Fig. 6. The BER result compares the Modified SLM with original OFDM, conventional SLM, DPP and SCS. Conventional SLM and DPP shown a worse BER performance when compared to the original OFDM. In many approaches, they were suggested to apply Forward Error Check (FEC) encoder and receiver at the transmitter and receiver respectively to overcome the BER performance degradation. However, in this paper, the application of FEC is not considered because it will increase the complexity of the system as well as leading to cost increment. Meanwhile, the modified SLM shown a better improvement when compared to the conventional one even though it slightly exceeded the original OFDM value.

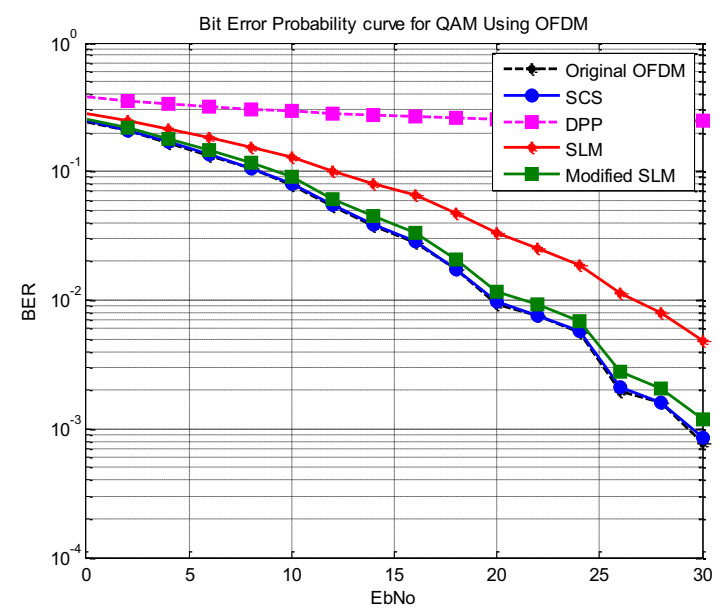

Figure 6. Comparison between BER of the Modified SLM, original OFDM, conventionalSLM, DPP and SCS.

The relationship between side information (SI) to the performance of BER is investigated by comparing the Modified SLM using bit SI and symbol SI. The bit SI which in the form of binary bits are embedded into the codeword sequence before the digital modulation process. Meanwhile, the symbol SI which in the form of complex number are embedded into the symbol sequence before signal transmissions. The BER performance of bit SI and symbol SI are shown in Fig. 7. As shown in Fig. 7, the symbol SI exceeded the original OFDM while bit SI indicates a similar performance as original OFDM. This finding explains that the symbol SI are tend to be interfered.

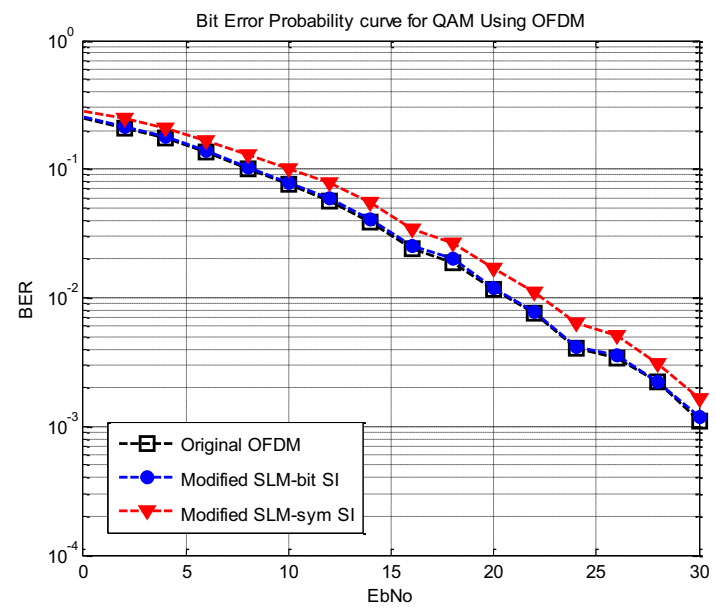

Figure 7. Comparison between BER of Modified Selective Mapping (SLM) using embedded bits SI and symbol SI.

\section{Conclusions}

Selective Mapping is widely used to reduce high PAPR in OFDM system in the cost of BER performance degradation. In this paper, a modified Selective Mapping technique based on circulant shift technique has been proposed to overcome the BER degradation that occurred 
using SLM and further reduce the high PAPR. Furthermore, theproposed embedded bits SIoffer a better performance of BERwhen to compare to symbol SI that had been used in conventional SLM.

\section{Acknowledgement}

We are grateful to the Universiti Teknologi Mara, UiTM for providing scholarship to Author 1. This paper is part of research work that supported by the Faculty of Electrical Engineering, Universiti Teknologi Mara, Shah Alam, Selangor Malaysia.

\section{References}

1. L. M. Correia, D. Zeller, O. Blume, D. Ferling, Y. Jading, I. Godor, G. Auer, and L. Van der Perre, Challenges and Enabling Technologies for Energy Aware Mobile Radio Networks, IEEE Signal Process. Mag., 48, 11, pp. 66-72 (2010).

2. S. H. Wang, J. C. Sie, C. P. Li, and Y. F. Chen, A Low-Complexity PAPR Reduction Scheme for OFDMA Uplink Systems, IEEE Trans. Wirel. Commun., 10, 4, pp. 1242-1251, Apr (2011).

3. M. Chauhan, Different Techniques to Reduce the PAPR in OFDM System, Int. J. Eng. Res. Appl., 2, 3, pp. 1292-1294 (2012).
4. H. B. Jeon, J. S. No, and D. J. Shin, A LowComplexity SLM Scheme Using Additive mapping Sequences for PAPR Reduction of OFDM Signals, IEEE Trans. Broadcast., 57, 4, pp. 866-875 (2011).

5. L. Ning, M. Yang, Z. Wang, and Q. Guo, A novel SLM method for PAPR reduction of OFDM system, in IEEE Vehicular Technology Conference (2012).

6. S. Y. Le Goff, S. S. Al-Samahi, B. K. Khoo, C. C. Tsimenidis, and B. S. Sharif, Selected mapping without side information for PAPR reduction in OFDM, IEEE Trans. Wirel. Commun., 8, 7, pp. 3320-3325 (2009).

7. A. Vallavaraj, B. G. Stewart, D. K. Harrison, and F. G. McIntosh, Reducing the PAPR of OFDM using a simplified scrambling SLM technique with no explicit side information, in Proceedings of the International Conference on Parallel and Distributed Systems - ICPADS, pp. 902-907(2008).

8. J. Wen, S. Lee, and C. Kung, SLM-Based Data Position Permutation Method for PAPR Reduction in OFDM Systems, Wirel. Commun. Mob. Comput., 13, 11, pp. 985-997 (2015).

9. F. Shamshad, U. Javed, and S. Saleem, Physical Layer Aspects of 3GPP s Long Term Evolution ( LTE ), Adv. Comput. Sci. its Appl., 2, 1, pp. 287294 (2012). 